Supporting Information:

\title{
A Silica Supported Molecular Palladium Catalyst for Selective Hydrodeoxygenation of Aromatic Compounds Under Mild Conditions
}

\author{
Nicholas A. DeLucia, ${ }^{\dagger}$ Amy Jystad,,+ Katherine Vander Laan,,+ John \\ Meynard M. Tengco, ${ }^{\S}$ Marco Caricato, ${ }^{*}$ Aaron K. Vannucci* ${ }^{*}$ \\ ${ }^{\dagger}$ University of South Carolina, Department of Chemistry and Biochemistry, \\ Columbia, SC USA 29208 \\ $\S$ University of South Carolina, Department of Chemical Engineering, Columbia, \\ SC USA 29208 \\ University of Kansas, Department of Chemistry, Lawrence, KS USA 66045
}

\section{Corresponding Author}

*E-mail: vannucci@mailbox.sc.edu,mcaricato@ku.edu

\section{Contents}

Prepared Catalyst - pg. S2

Pre-Reaction Characterization - pg. S2-S3

Kinetic Analysis - pg. S3-S5

Post-Reaction Characterization and Recyclability - pg. S5-S7

NMR Spectra of $\mathbf{1}-$ pg. S8-S9

Computational Data - pg. S10-S22 


\section{Prepared Catalyst}

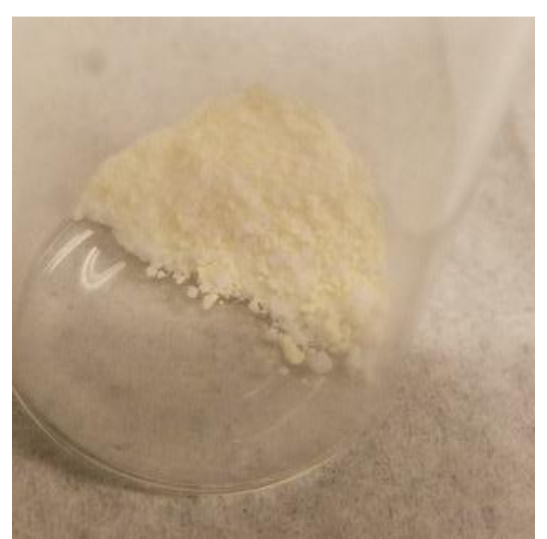

Figure S1. Image of prepared 1-SiO $\mathbf{S}_{2}$ following $\mathrm{MeOH}$ washing and subsequent drying.

\section{Pre-Reaction Characterization}

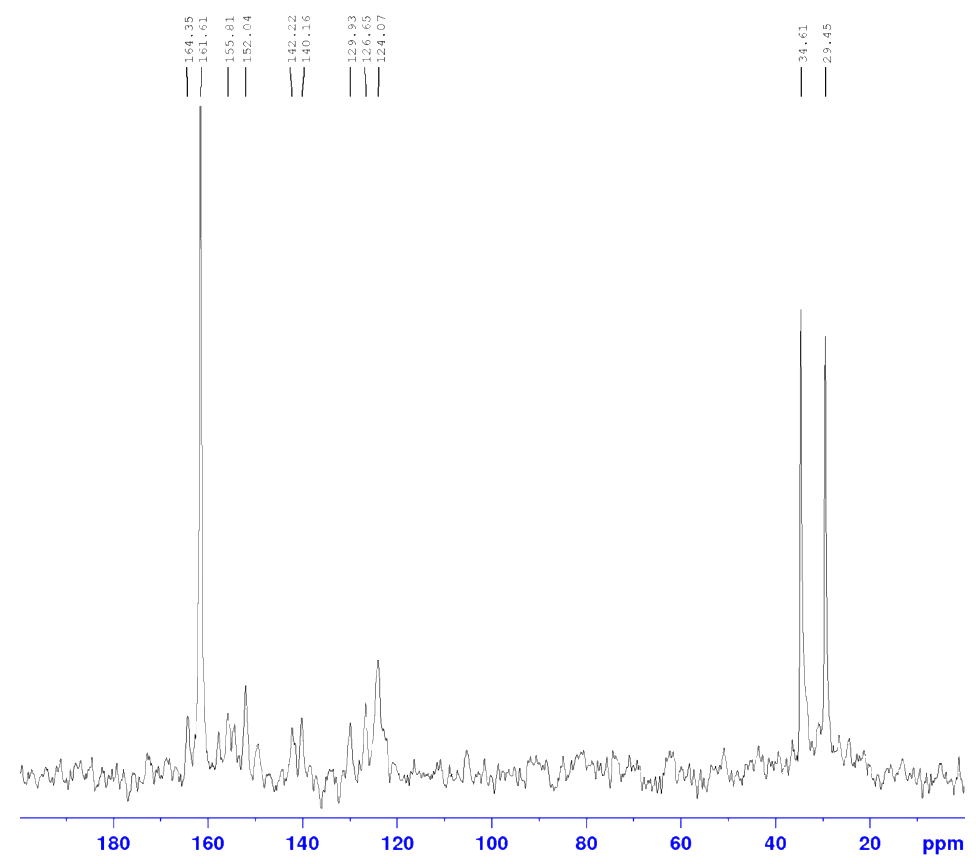

Figure S2. ${ }^{13} \mathrm{C}$ NMR spectra of $\mathbf{1 - S i O}$, peaks located at 161.61, 34.61, and 29.45 correspond to residual DMF 


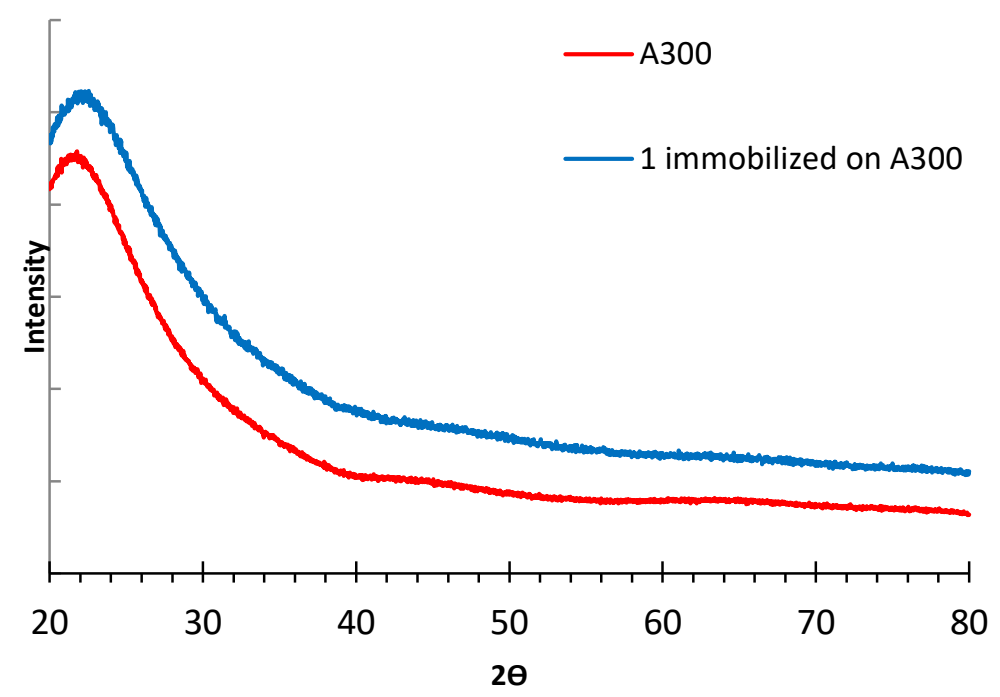

Figure S3. Powder X-ray diffraction profiles of fresh A300 (red), and as prepared 1-SiO 2 (blue).

\section{Kinetic Analysis}

$$
\begin{array}{ll}
\text { rate }=\frac{\Delta[\mathrm{tol}]}{\mathrm{t}}=k[\mathrm{BA}]\left[P d_{\text {int }}\right] & {[\mathrm{Pd}]_{\text {tot }}=\frac{k_{-1}\left[P d_{\text {int }}\right]}{k_{1}\left[\mathrm{H}_{2}\right]}+\left[P d_{\text {int }}\right]} \\
{[\mathrm{Pd}]_{\text {tot }}=\left[\mathrm{Pd}^{\mathrm{II}}\right]+\left[P d_{\text {int }}\right]} & {[\mathrm{Pd}]_{\text {tot }}=\left[P d_{\text {int }}\right]\left(1+\frac{k_{-1}}{k_{1}\left[\mathrm{H}_{2}\right]}\right)} \\
\mathrm{K}=\frac{k_{1}}{k_{-1}}=\frac{\left[P d_{\text {int }}\right]}{\left[\mathrm{Pd}^{\mathrm{II}}\right]\left[\mathrm{H}_{2}\right]} & \frac{k_{-1}}{k_{1}\left[\mathrm{H}_{2}\right]} \cong 0 \text { at high }\left[\mathrm{H}_{2}\right] \text { and large } \mathrm{k}_{1} \text { (assumed) } \\
{\left[\mathrm{Pd}^{\mathrm{II}}\right]=\frac{k_{-1}\left[P d_{\text {int }}\right]}{k_{1}\left[\mathrm{H}_{2}\right]}} & {[\mathrm{Pd}]_{\text {tot }} \cong\left[P d_{\text {int }}\right]} \\
& \text { rate }=k[\mathrm{BA}][\mathrm{Pd}]_{\text {tot }}
\end{array}
$$

Figure S4. Kinetic derivation to determine the rate law for the 1-SiO $\mathbf{S}_{2}$ catalyzed $\mathrm{HDO}$ of benzyl alcohol (BA) to toluene (tol) 


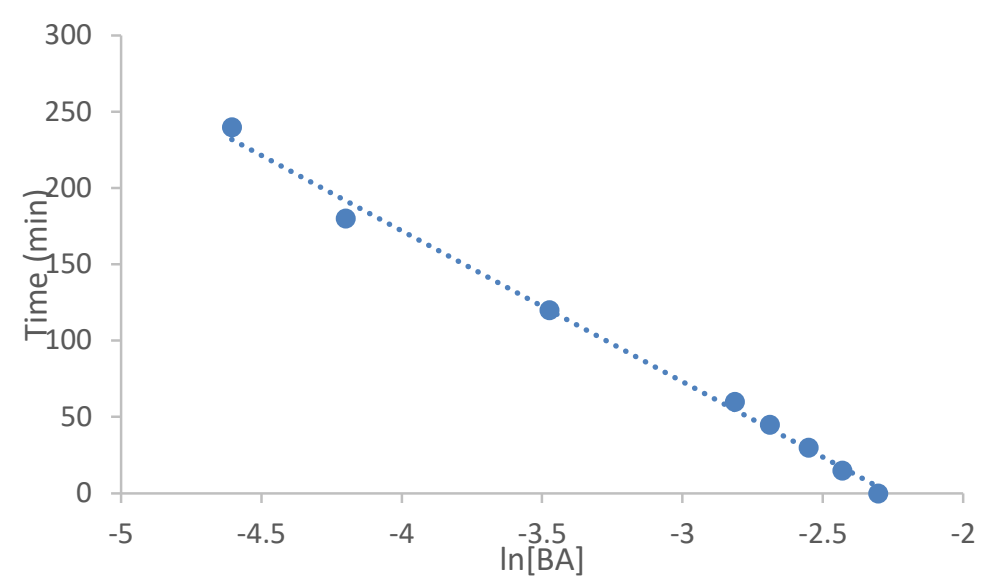

Figure S5. Plot of $\ln [\mathrm{BA}]$ vs. time showing a linear fit

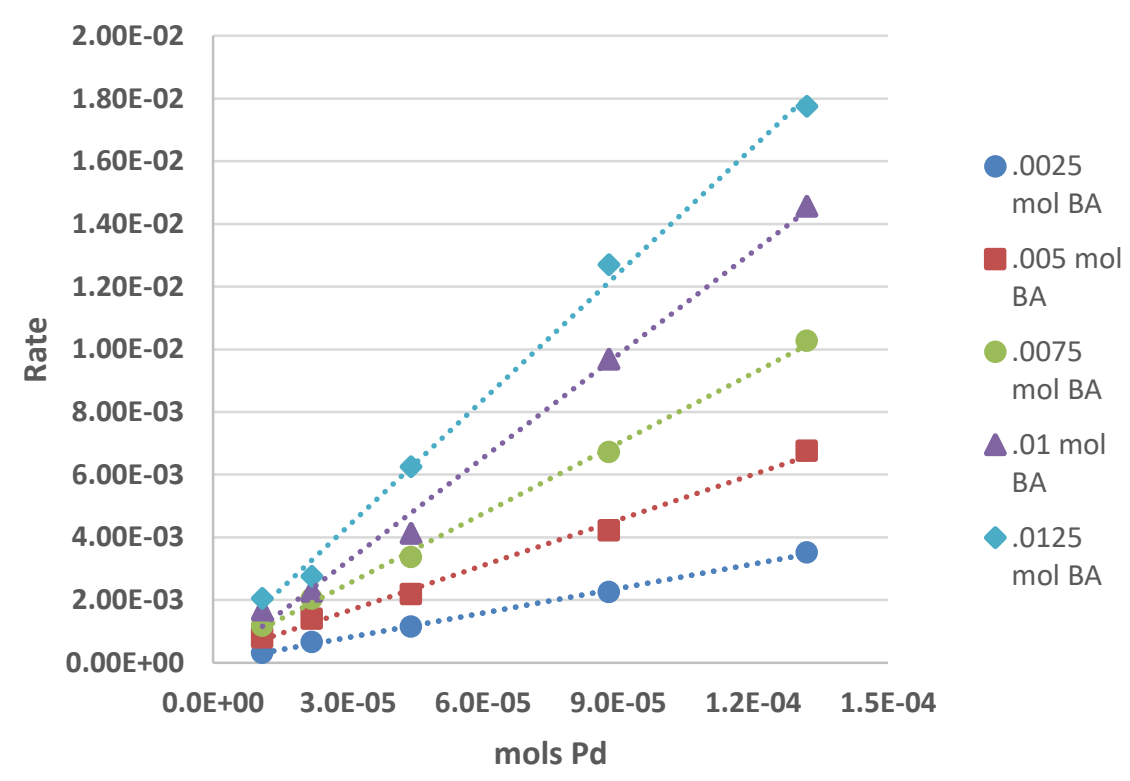

Figure S6. Plot of mols Pd vs. the initial rates of reaction (15 min) at varying amount of $\mathrm{BA}$ 


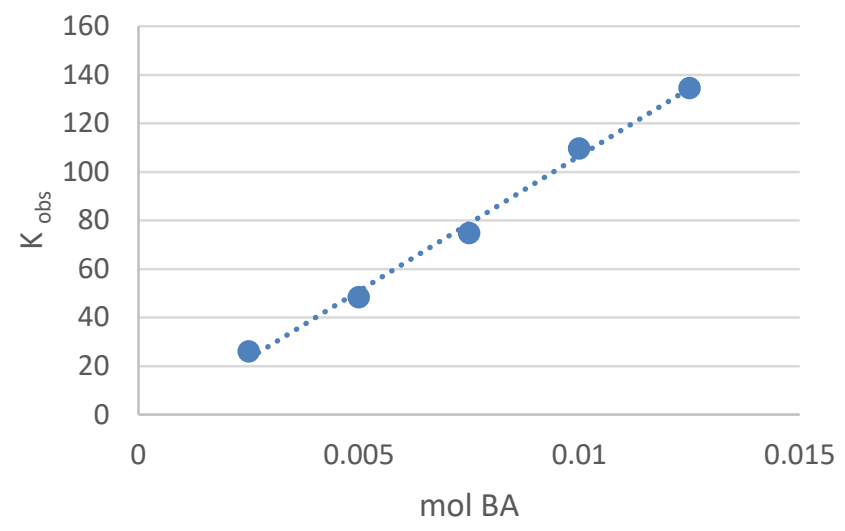

Figure S7. Plot of mols BA vs. the $k_{\text {obs }}$ values derived from the slopes of the plots of Figure S6, showing a linear relationship

\section{Post Reaction Characterization \& Recyclability}

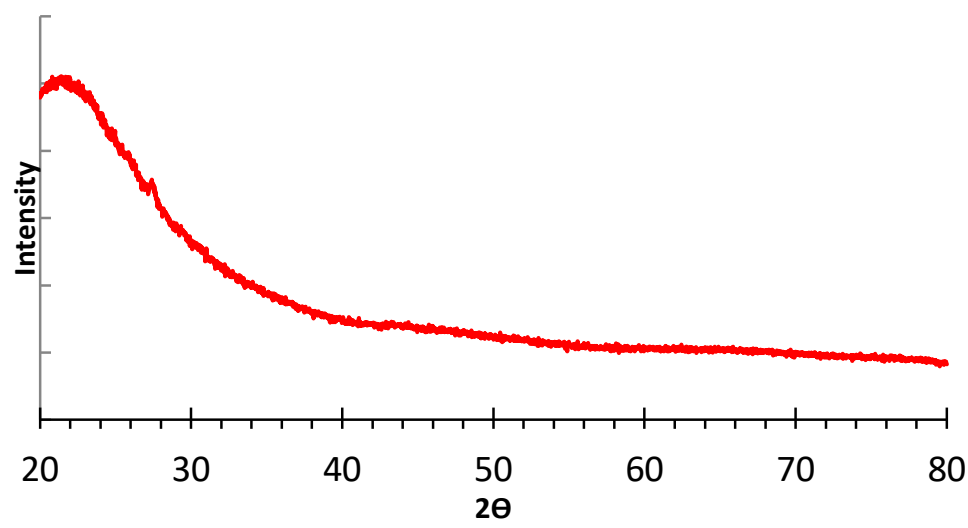

Figure S8. Powder X-ray diffraction profile of $\mathbf{1 - S i O} 2$ following the low conversion, 30-minute, $100{ }^{\circ} \mathrm{C}$ catalytic reactions (red) in dodecane. No signals for metallic Pd peaks are present. 
Table S1. Summary of recycled catalytic and pre-treated results of $\mathbf{1 - S i O}_{2}$ in dodecane. ${ }^{\mathrm{a}}$

\begin{tabular}{ccccc}
\hline Rxn Number & Convers. (\%) & $\begin{array}{c}\text { Select. to } \\
\text { Toluene (\%) }\end{array}$ & $\begin{array}{c}\text { Select. to } \\
\text { methyl } \\
\text { cyclohexane } \\
(\%)\end{array}$ & $\begin{array}{c}\text { Select. to } \\
\text { cyclohexane } \\
\text { methanol (\%) }\end{array}$ \\
\hline 1 & $>99$ & $>99$ & 0 & 0 \\
2 & $>99$ & 91 & 7 & 2 \\
3 & $>99$ & 95 & 3 & 2 \\
4 & $>99$ & 92 & 4 & 4 \\
5 & $>99$ & 92 & 4 & 4 \\
\hline
\end{tabular}

${ }^{a} 8.8 \times 10^{-5} \mathrm{~mol} \mathbf{1}\left(450 \mathrm{mg}\right.$ total $\mathbf{1 - S i O}{ }_{2}$, rxn. 1$)$ in $25 \mathrm{~mL}$ of solvent with $0.1 \mathrm{M}$ benzyl alcohol. Reaction time 4 hrs.

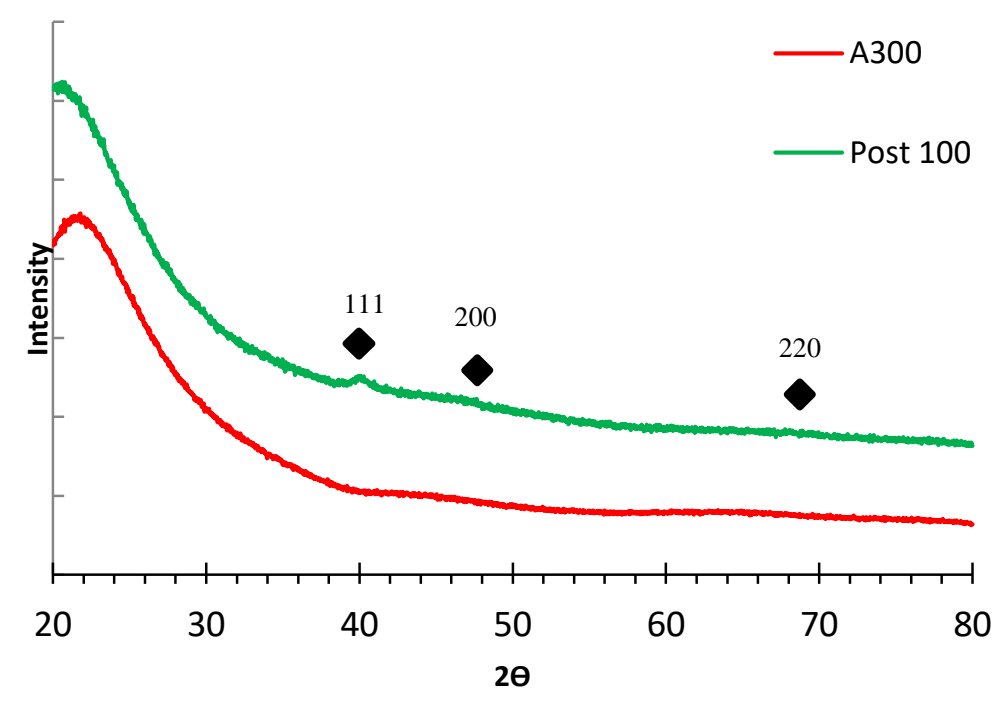

Figure S9. Power X-ray diffraction profiles of fresh A300 (red) and 1$\mathrm{SiO}_{2}$ following full conversion, recycle studies at $100{ }^{\circ} \mathrm{C}$ reaction (green) in dodecane. Metallic Pd peak positions denoted with a diamond with crystal planes denoted above. 

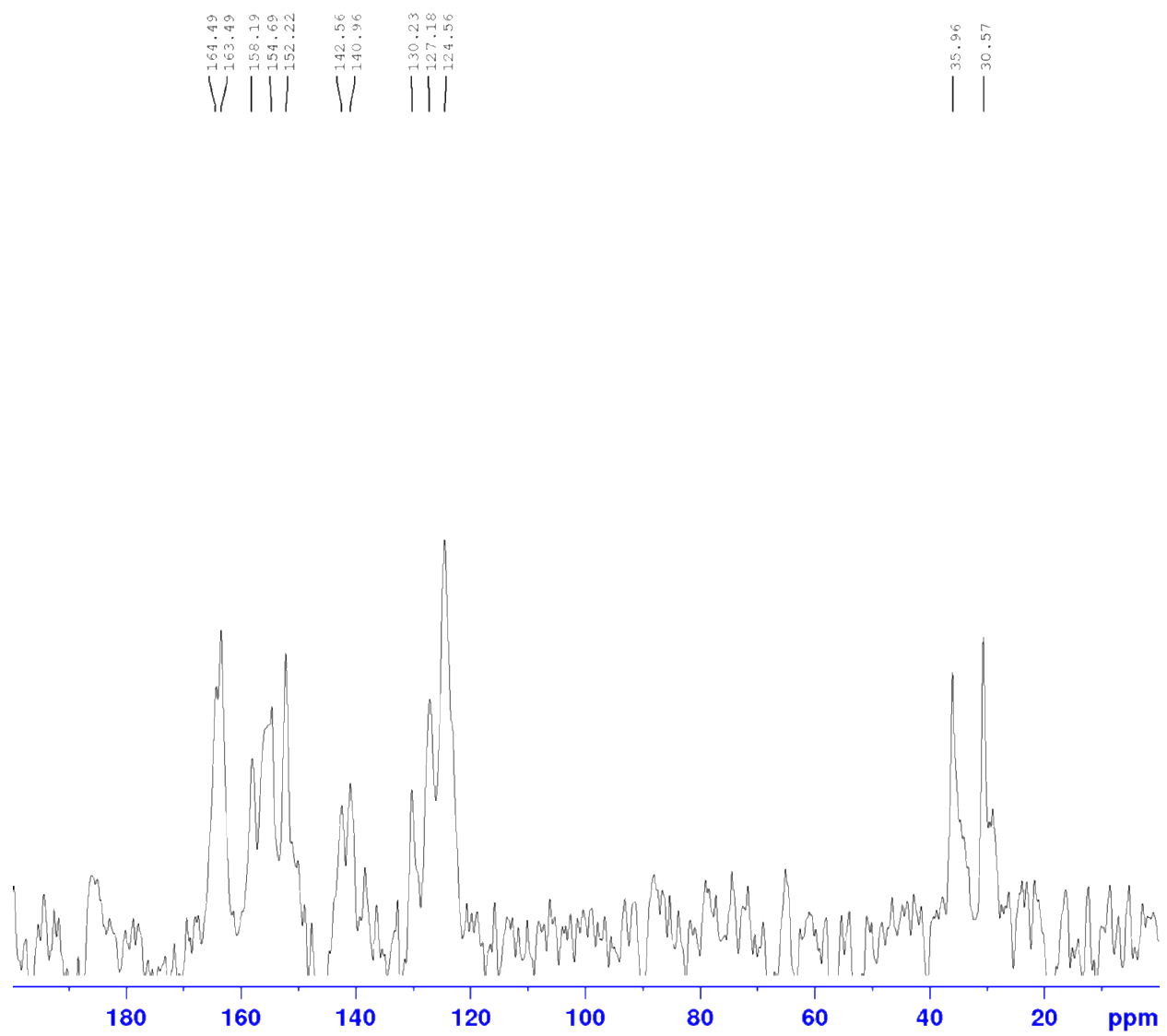

Figure S10. ${ }^{13} \mathrm{C}$ NMR spectra of $\mathbf{1 - S i O} \mathbf{S}_{2}$ following a reaction in dodecane. Small amount of residual DMF are present and easily identified at $163.49,35.96$ and 30.57 . 


\section{Solution-phase NMR}

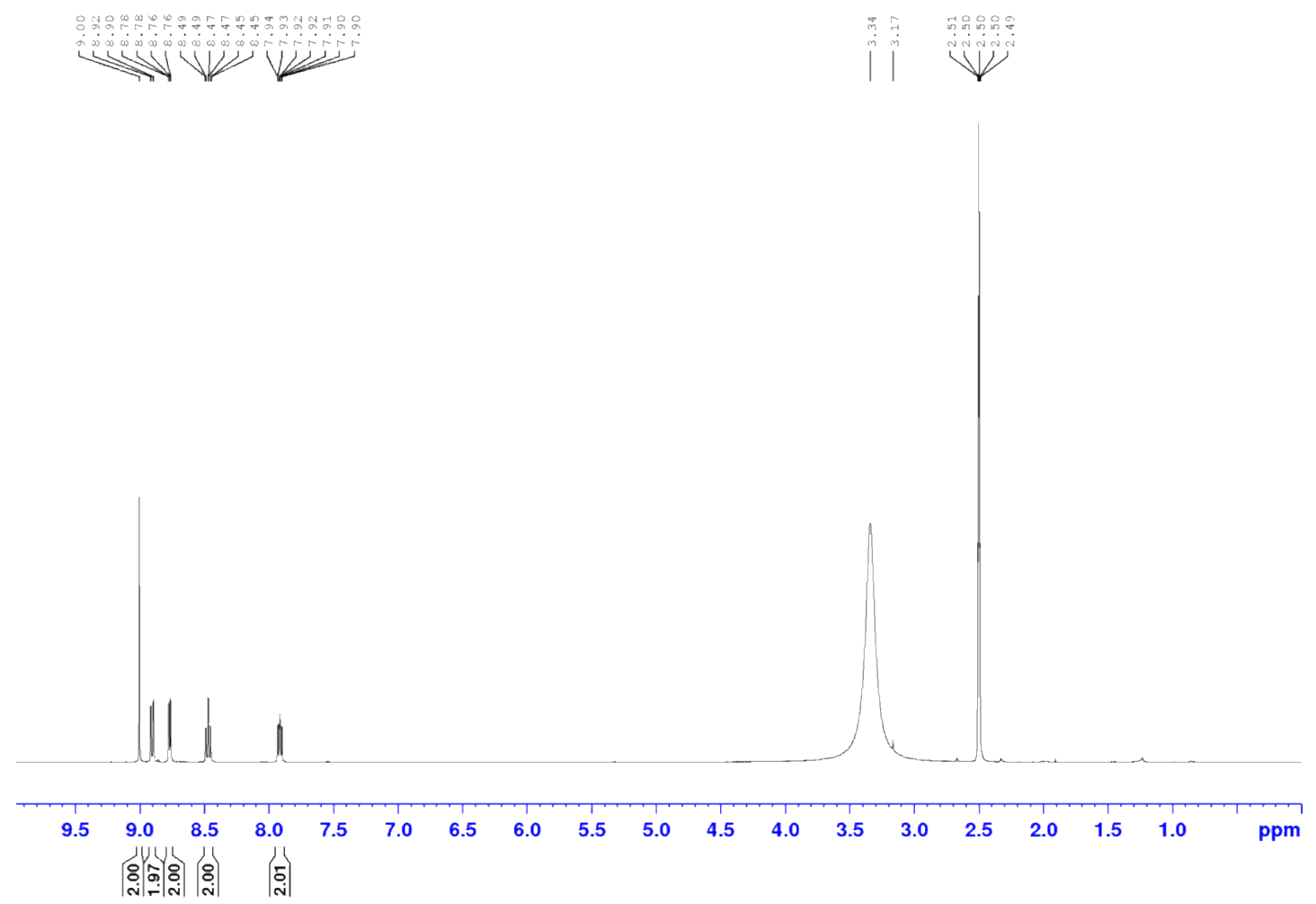

Figure S11. ${ }^{1} \mathrm{H}$ NMR spectra of $\mathbf{1}$, broad singlet at $3.34 \mathrm{ppm}$ is $-\mathrm{COOH}$ of $\mathbf{1}$ exchanging with water present in DMSO- $\mathrm{d}_{6}$ 


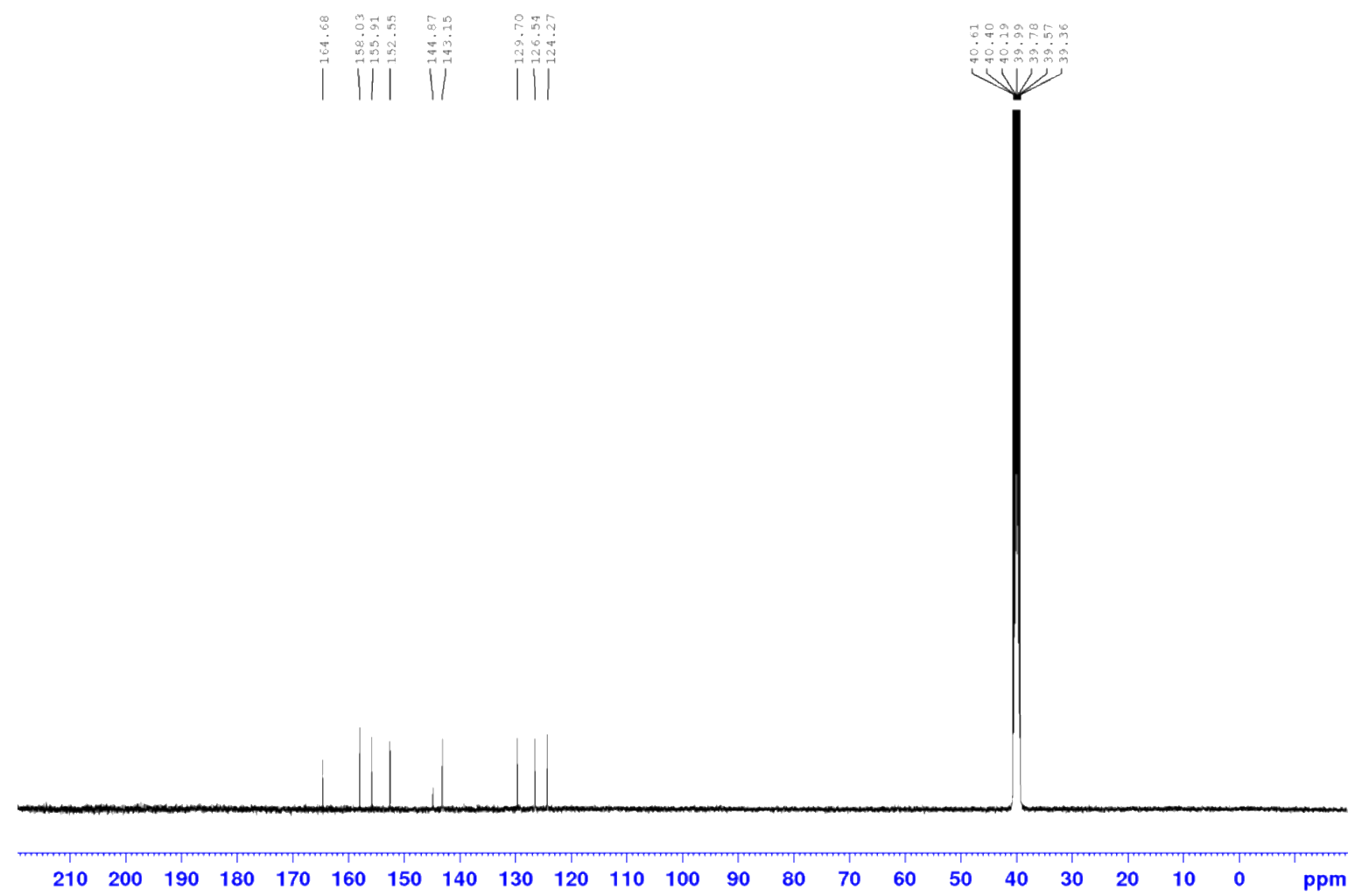

Figure S12. ${ }^{13} \mathrm{C}$ NMR of 1 in DMSO-d 6 


\section{Computational Data}

Table S2. Rigid IE $\left(\mathrm{kcal} \mathrm{mol}^{-1}\right)$ of all twenty-five sites in contour plot, beginning in bottom left corner and proceeding vertically on the plot. IE are for complex $\mathbf{1}$ and $\mathbf{2}$, in vacuo and in DMF.

\begin{tabular}{|c|c|c|}
\hline Site & $\begin{array}{l}\text { IE of complex } 1 \\
\text { in vacuo }\end{array}$ & $\begin{array}{l}\text { IE of complex } 1 \\
\text { in DMF }\end{array}$ \\
\hline 1 & -24.6 & -10.2 \\
\hline 2 & -15.1 & -7.7 \\
\hline 3 & -29.0 & -10.0 \\
\hline 4 & -12.0 & 18.4 \\
\hline 5 & -20.1 & -9.6 \\
\hline 6 & -26.9 & 2.7 \\
\hline 7 & -16.4 & -6.6 \\
\hline 8 & -23.0 & -0.1 \\
\hline 9 & -28.6 & -12.6 \\
\hline 10 & -29.4 & -9.6 \\
\hline 11 & -47.0 & -14.2 \\
\hline 12 & -19.5 & -10.4 \\
\hline 13 & -14.2 & -4.6 \\
\hline 14 & -17.0 & -7.1 \\
\hline 15 & -3.0 & -6.9 \\
\hline 16 & -23.8 & -8.3 \\
\hline 17 & -33.4 & -13.9 \\
\hline 18 & -18.5 & -9.0 \\
\hline 19 & -3.8 & -8.7 \\
\hline 20 & -13.8 & -6.6 \\
\hline 21 & -4.2 & -6.3 \\
\hline 22 & -19.4 & -11.5 \\
\hline 23 & 8.7 & -3.0 \\
\hline 24 & -12.7 & -7.5 \\
\hline 25 & -26.4 & -9.9 \\
\hline
\end{tabular}


Table S3. IE ( $\mathrm{kcal} \mathrm{mol}^{-1}$ ) for Sites A-D in DMF before (Rigid) and after (Relaxed) complex relaxation, either protonated or deprotonated. Also includes IE of single point calculations (Single Pt) of the final relaxed geometries with and without $\mathrm{Pd}$ and $\mathrm{Cl}$.

\begin{tabular}{cccccccc}
\hline Site & $\begin{array}{c}\mathrm{PdtpyCOOH}^{2+} \\
\text { Rigid }\end{array}$ & $\begin{array}{c}\text { PdtpyCOOHCl } \\
\text { Relaxed }\end{array}$ & $\begin{array}{c}\mathrm{PdtpyCOOH}^{2+} \\
\text { Single Pt }\end{array}$ & $\begin{array}{c}\text { tpyCOOH } \\
\text { Single Pt }\end{array}$ & $\begin{array}{c}\text { PdtpyCOOCl }^{2} \\
\text { Relaxed }\end{array}$ & $\begin{array}{c}\text { PdtpyCOO }^{1+} \\
\text { Single Pt }\end{array}$ & $\begin{array}{c}\text { tpyCOO }^{1-} \\
\text { Single Pt }\end{array}$ \\
\hline A & -14 & -26 & -30 & -30 & -25 & -25 & -18 \\
B & -10 & -22 & -26 & -27 & -19 & -19 & -10 \\
C & -13 & -26 & -29 & -33 & -35 & -33 & -32 \\
D & -14 & -31 & -34 & -34 & -30 & -28 & -21 \\
\hline
\end{tabular}

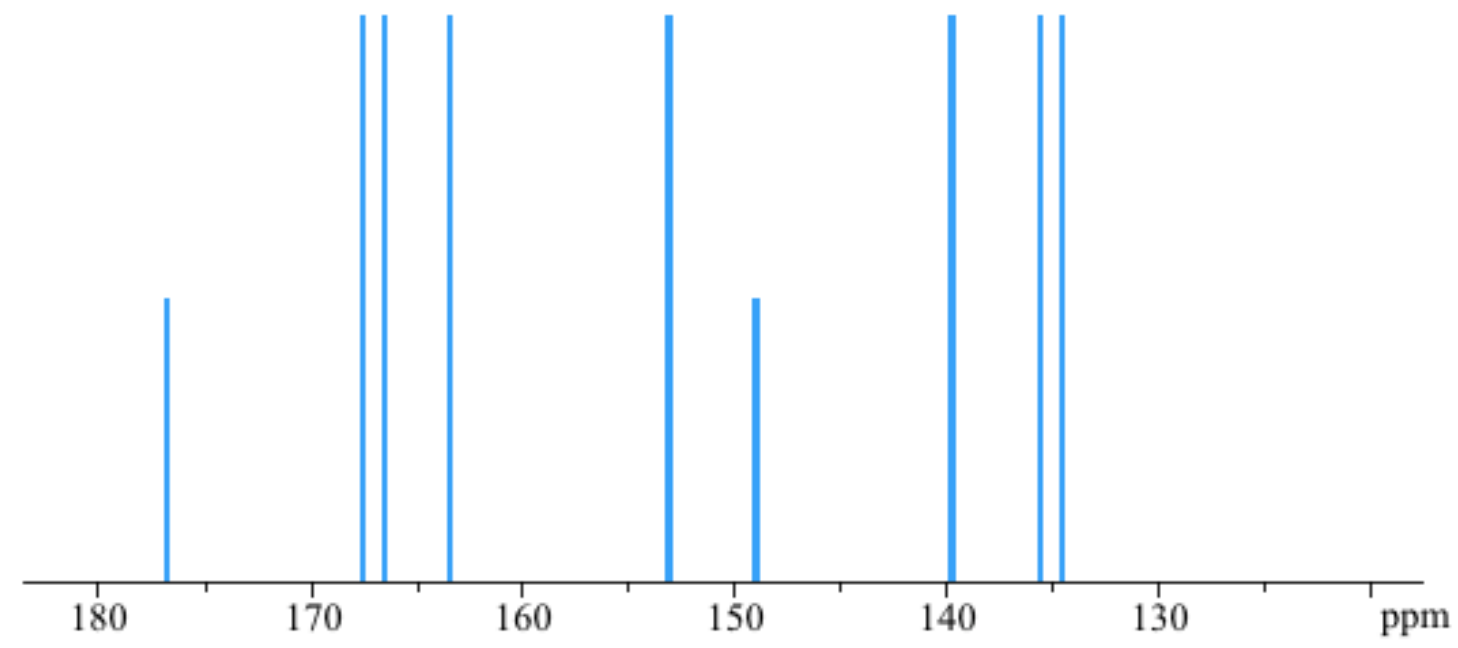

$\mathrm{A} \square \mathrm{B} \square \mathrm{C} \square \mathrm{D}$

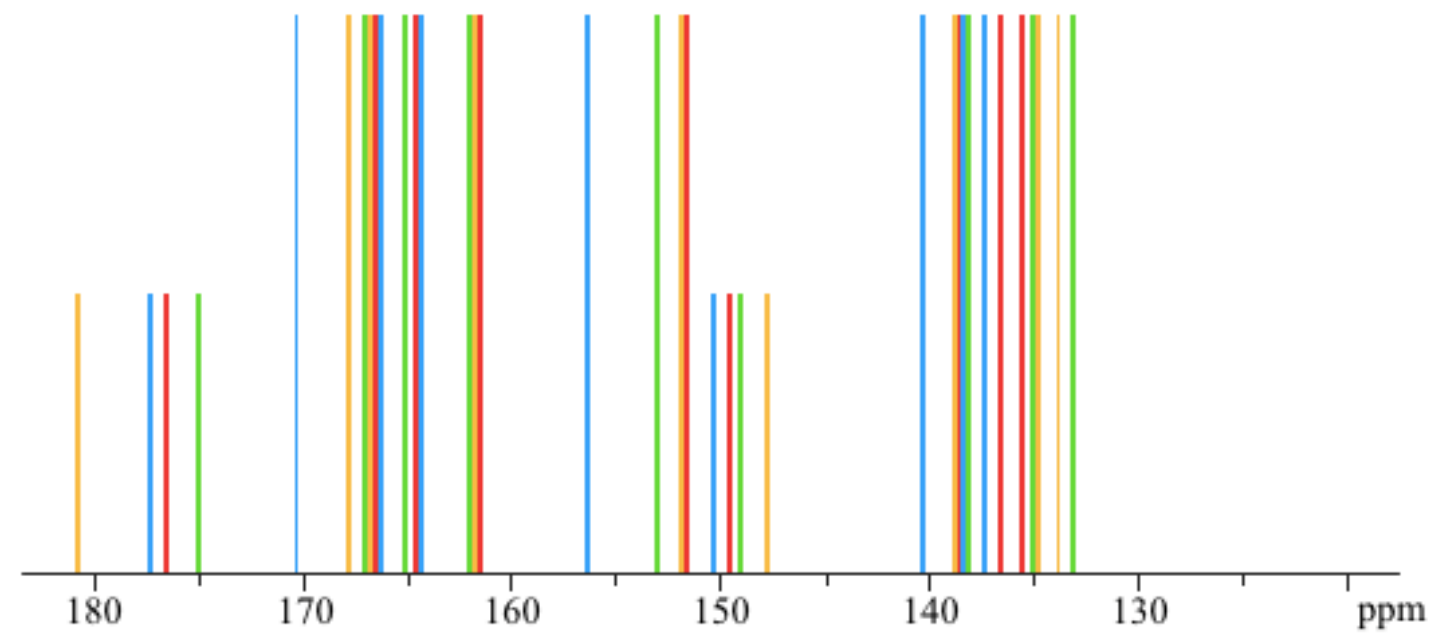

Figure S13. Simulated ${ }^{13} \mathrm{C}$ NMR spectra of 1 in solution (top) and on the silica surface (bottom). A-D refer to the four sites studied in the main text. All calculations are performed using implicit DMSO solvent. 


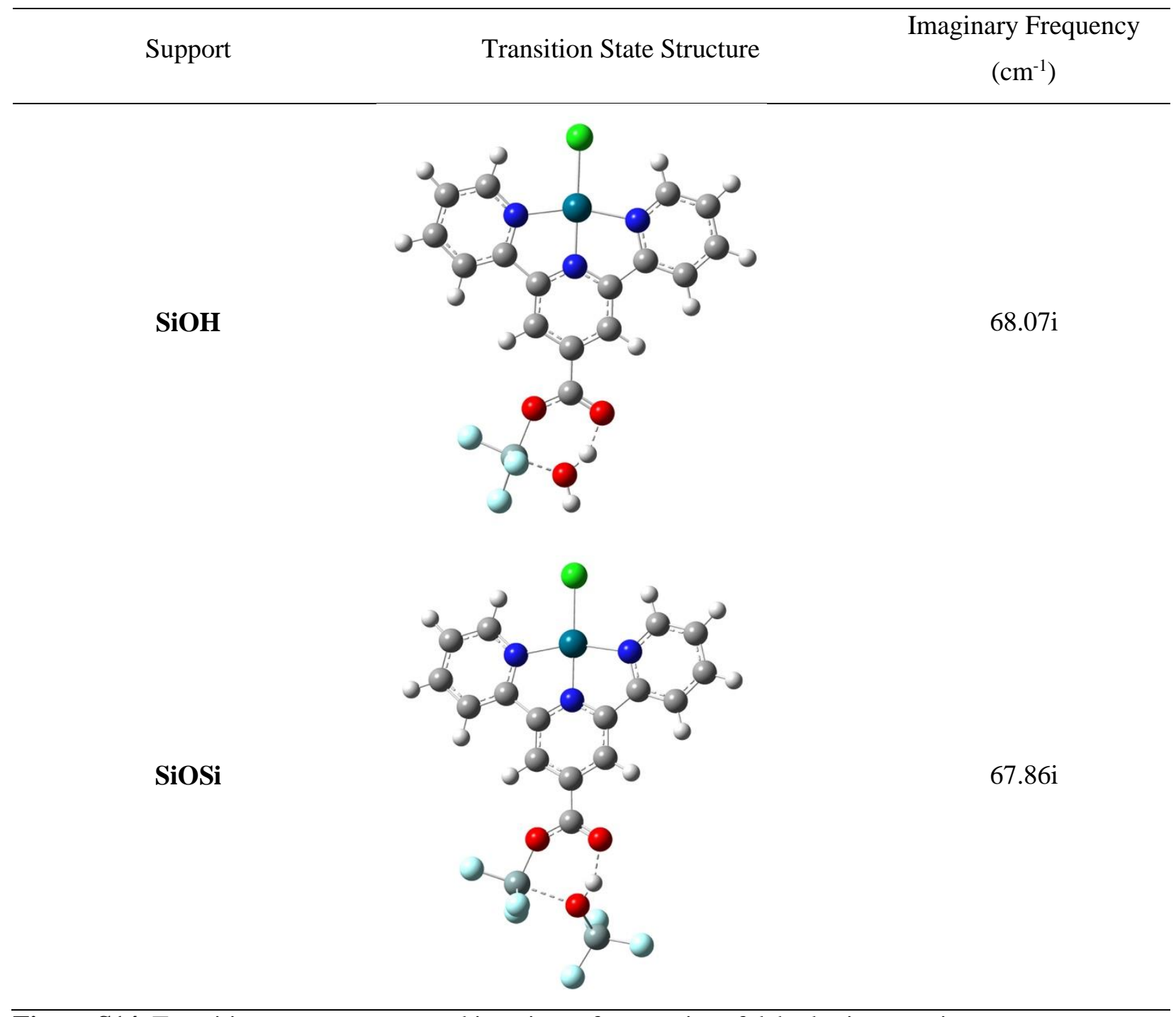

Figure S14. Transition state structure and imaginary frequencies of dehydration reaction. 


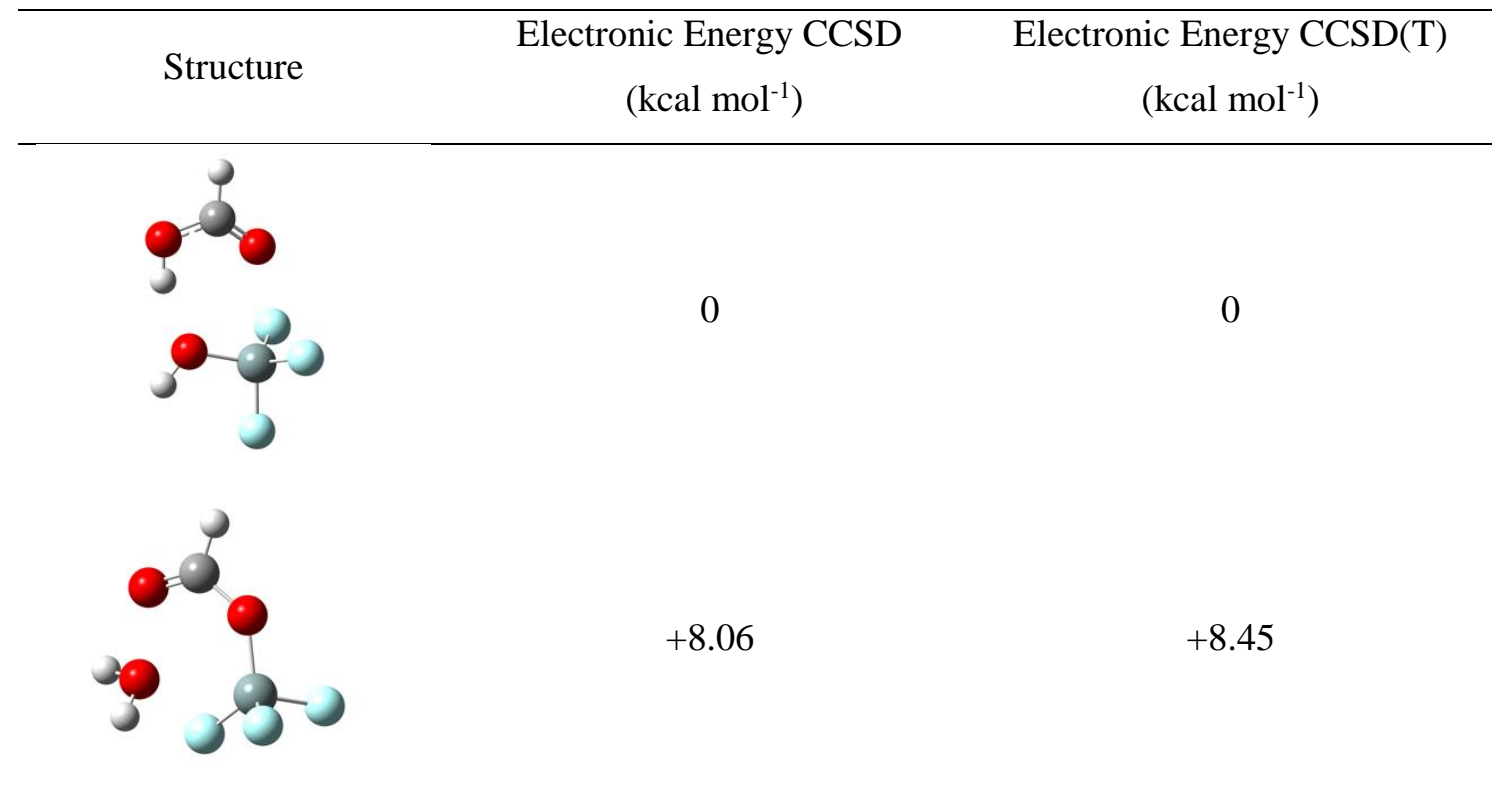

Figure S15. Structure and relative energies $\left(\mathrm{kcal} \mathrm{mol}^{-1}\right)$ of hydrogen and covalently bonded formic acid on a silanol computed at CCSD and CCSD(T) level. 


\begin{tabular}{|c|c|c|c|}
\hline \multicolumn{4}{|c|}{ Cartesian coordinates of $1(\AA)$ on Site $A$} \\
\hline $\mathrm{C}$ & 3.23784800 & -17.37590200 & 15.36219400 \\
\hline $\mathrm{C}$ & 2.91261700 & -18.69231400 & 15.01606400 \\
\hline $\mathrm{C}$ & 4.57238200 & -16.96034400 & 15.42033900 \\
\hline $\mathrm{C}$ & 3.94560700 & -19.56948800 & 14.68980400 \\
\hline $\mathrm{H}$ & 1.88259400 & -19.01565600 & 14.96565100 \\
\hline $\mathrm{C}$ & 5.57126200 & -17.88582800 & 15.12175600 \\
\hline $\mathrm{H}$ & 4.81359200 & -15.93568900 & 15.67534000 \\
\hline $\mathrm{N}$ & 5.21798800 & -19.13609200 & 14.76133400 \\
\hline $\mathrm{C}$ & 3.81632000 & -20.93847700 & 14.15453000 \\
\hline $\mathrm{C}$ & 7.02849200 & -17.66271100 & 15.12077100 \\
\hline $\mathrm{Pd}$ & 6.63859800 & -20.39687500 & 14.31516800 \\
\hline $\mathrm{C}$ & 2.60403500 & -21.52921900 & 13.81094200 \\
\hline $\mathrm{N}$ & 5.00426000 & -21.57744500 & 13.90785100 \\
\hline $\mathrm{C}$ & 7.63056300 & -16.44695200 & 15.42459300 \\
\hline $\mathrm{N}$ & 7.78256400 & -18.75565400 & 14.77976400 \\
\hline $\mathrm{C}$ & 2.60607900 & -22.77962400 & 13.18740900 \\
\hline $\mathrm{H}$ & 1.67112000 & -21.01021200 & 13.99513900 \\
\hline $\mathrm{C}$ & 5.00525500 & -22.78457800 & 13.32847600 \\
\hline $\mathrm{C}$ & 9.02208700 & -16.34512700 & 15.37507200 \\
\hline $\mathrm{H}$ & 7.02218000 & -15.59086500 & 15.69193700 \\
\hline $\mathrm{C}$ & 9.11677100 & -18.65636500 & 14.73312900 \\
\hline $\mathrm{C}$ & 3.82047700 & -23.41507900 & 12.94308200 \\
\hline $\mathrm{H}$ & 1.67296600 & -23.23792900 & 12.87626500 \\
\hline $\mathrm{H}$ & 5.97640300 & -23.23409500 & 13.15998800 \\
\hline $\mathrm{C}$ & 9.77466000 & -17.46180200 & 15.02471900 \\
\hline $\mathrm{H}$ & 9.50539300 & -15.40173000 & 15.60746800 \\
\hline $\mathrm{H}$ & 9.65400000 & -19.55524700 & 14.45550600 \\
\hline $\mathrm{H}$ & 3.86598600 & -24.38098500 & 12.45295600 \\
\hline $\mathrm{H}$ & 10.85706000 & -17.42467100 & 14.97353800 \\
\hline $\mathrm{C}$ & 2.16645600 & -16.34824900 & 15.57762200 \\
\hline $\mathrm{O}$ & 2.38114400 & -15.18797100 & 15.86740100 \\
\hline $\mathrm{O}$ & 0.93600700 & -16.85197000 & 15.37062200 \\
\hline $\mathrm{O}$ & -1.22448300 & -15.80352800 & 14.18421200 \\
\hline $\mathrm{H}$ & -2.03470500 & -15.82427100 & 14.71451500 \\
\hline $\mathrm{O}$ & -5.23077000 & -13.84133300 & 14.45233300 \\
\hline $\mathrm{H}$ & -6.12610100 & -14.03823300 & 14.76020800 \\
\hline $\mathrm{O}$ & 0.42007100 & -16.71347700 & 12.57989400 \\
\hline $\mathrm{H}$ & 0.45204100 & -17.05420600 & 13.48568200 \\
\hline $\mathrm{O}$ & 2.45916100 & -18.34986700 & 12.18062700 \\
\hline $\mathrm{Si}$ & 3.58268200 & -18.86063100 & 11.08012100 \\
\hline $\mathrm{Si}$ & -6.67958600 & -17.27114700 & 12.65318400 \\
\hline $\mathrm{Si}$ & -1.56064900 & -15.05316900 & 12.70377700 \\
\hline $\mathrm{Si}$ & -4.76012300 & -14.63845000 & 13.08362900 \\
\hline $\mathrm{Si}$ & 1.74877600 & -16.90806700 & 11.67662900 \\
\hline $\mathrm{Si}$ & 4.27265000 & -18.58479100 & 7.72249900 \\
\hline $\mathrm{Si}$ & 1.74888400 & -17.99243200 & 8.86873900 \\
\hline $\mathrm{Si}$ & 6.15587000 & -19.52903500 & 10.48774100 \\
\hline $\mathrm{Si}$ & -4.65961600 & -19.32426500 & 11.11080700 \\
\hline $\mathrm{Si}$ & -0.69291300 & -13.72060000 & 7.24219200 \\
\hline $\mathrm{Si}$ & -0.33372700 & -19.48055800 & 6.85840400 \\
\hline $\mathrm{Si}$ & -1.99370200 & -16.73044300 & 7.19056600 \\
\hline $\mathrm{Si}$ & 0.07939000 & -22.95054600 & 9.40061100 \\
\hline $\mathrm{Si}$ & 2.83551200 & -20.96930300 & 8.95722200 \\
\hline $\mathrm{Si}$ & 4.02686800 & -15.70838000 & 10.73049700 \\
\hline $\mathrm{Si}$ & 0.51394200 & -12.48711400 & 12.09661800 \\
\hline $\mathrm{Si}$ & -2.94846700 & -16.61517700 & 10.22263300 \\
\hline $\mathrm{Si}$ & -4.88844200 & -14.02773900 & 10.12625700 \\
\hline
\end{tabular}




\begin{tabular}{|c|c|c|c|}
\hline $\mathrm{Si}$ & -3.11664400 & -22.11082000 & 10.39093500 \\
\hline $\mathrm{Si}$ & 1.38198000 & -12.53686300 & 9.07682400 \\
\hline $\mathrm{O}$ & 1.64980000 & -11.69730000 & 13.12860000 \\
\hline $\mathrm{O}$ & -0.36098400 & -13.89032700 & 12.52902100 \\
\hline $\mathrm{O}$ & 1.23964300 & -17.01017800 & 10.09102200 \\
\hline $\mathrm{O}$ & -5.68950100 & -18.62347500 & 12.30679900 \\
\hline $\mathrm{O}$ & 1.30818100 & -21.75570100 & 9.17268600 \\
\hline $\mathrm{O}$ & 3.20304000 & -15.11900000 & 9.46851000 \\
\hline $\mathrm{O}$ & -0.77966600 & -11.50450000 & 11.90200000 \\
\hline $\mathrm{O}$ & 5.42798000 & -14.74200000 & 10.69450000 \\
\hline $\mathrm{O}$ & 3.24428900 & -19.88336500 & 7.61790200 \\
\hline $\mathrm{O}$ & 1.35010200 & -12.77013500 & 10.71018600 \\
\hline $\mathrm{O}$ & -5.54610900 & -16.11658200 & 13.12563200 \\
\hline $\mathrm{O}$ & 0.58536000 & -18.81970400 & 8.08251600 \\
\hline $\mathrm{O}$ & -1.38975000 & -22.41193800 & 10.17572500 \\
\hline $\mathrm{O}$ & -4.11113000 & -15.47399900 & 9.95159300 \\
\hline $\mathrm{O}$ & 2.86072700 & -15.68037100 & 11.93003500 \\
\hline $\mathrm{O}$ & 5.01240500 & -19.44613400 & 11.68109200 \\
\hline $\mathrm{O}$ & 3.05569300 & -17.42668500 & 8.01184000 \\
\hline $\mathrm{O}$ & -3.11157000 & -14.58756700 & 13.13164500 \\
\hline $\mathrm{O}$ & -2.13336100 & -16.93890000 & 8.82855000 \\
\hline $\mathrm{O}$ & 5.43947100 & -19.03274900 & 8.95001300 \\
\hline $\mathrm{O}$ & 0.49537800 & -13.74166900 & 8.38552200 \\
\hline $\mathrm{O}$ & 2.45424300 & -19.20045100 & 9.78049400 \\
\hline $\mathrm{O}$ & 3.46411800 & -20.80684200 & 10.66434000 \\
\hline $\mathrm{O}$ & -3.92479000 & -12.81860000 & 9.41964000 \\
\hline $\mathrm{O}$ & -1.90001700 & -15.99574100 & 11.36167600 \\
\hline $\mathrm{O}$ & -3.64299200 & -20.72944100 & 11.32902700 \\
\hline $\mathrm{O}$ & 4.21982300 & -17.34671600 & 10.57914200 \\
\hline $\mathrm{O}$ & -3.58624000 & -16.65910000 & 6.66480000 \\
\hline $\mathrm{O}$ & -2.03482000 & -12.88860000 & 7.81582000 \\
\hline $\mathrm{O}$ & -1.18875000 & -18.04305600 & 6.48431600 \\
\hline $\mathrm{O}$ & -1.15542900 & -15.30529000 & 6.92444500 \\
\hline $\mathrm{O}$ & -5.10556400 & -13.75700700 & 11.74773300 \\
\hline $\mathrm{O}$ & -3.60110000 & -21.64100000 & 8.89079000 \\
\hline $\mathrm{O}$ & -3.66193200 & -18.05607100 & 10.68115100 \\
\hline $\mathrm{O}$ & -1.57261000 & -20.58140000 & 7.34864000 \\
\hline $\mathrm{O}$ & -5.81157000 & -19.83540000 & 10.02000000 \\
\hline $\mathrm{H}$ & -4.10224700 & -22.27191500 & 8.36887300 \\
\hline $\mathrm{H}$ & -5.72505900 & -19.32767000 & 9.20986000 \\
\hline $\mathrm{H}$ & -1.76387200 & -21.32818000 & 6.77650500 \\
\hline $\mathrm{H}$ & 4.03242800 & -21.50775100 & 10.99198400 \\
\hline $\mathrm{H}$ & 5.66798400 & -14.85687400 & 9.77211100 \\
\hline $\mathrm{H}$ & 3.77534200 & -14.93382000 & 8.72032700 \\
\hline $\mathrm{H}$ & -0.62093500 & -10.59356500 & 11.64392500 \\
\hline $\mathrm{H}$ & 2.19582800 & -11.01809600 & 12.72593900 \\
\hline $\mathrm{H}$ & -2.22286200 & -12.05336100 & 7.38152100 \\
\hline $\mathrm{H}$ & -4.42114200 & -12.05134900 & 9.12542600 \\
\hline $\mathrm{H}$ & -3.81246800 & -16.30819000 & 5.80034500 \\
\hline $\mathrm{O}$ & -7.90372000 & -17.71325700 & 13.98090900 \\
\hline $\mathrm{H}$ & -8.77455100 & -17.47351900 & 13.65567300 \\
\hline $\mathrm{O}$ & -7.41451000 & -16.50733300 & 11.25914800 \\
\hline $\mathrm{H}$ & -8.36850000 & -16.49966700 & 11.36612800 \\
\hline $\mathrm{O}$ & -3.96632700 & -23.55849600 & 10.96690900 \\
\hline $\mathrm{H}$ & -4.04730700 & -24.16481600 & 10.22703100 \\
\hline $\mathrm{O}$ & 0.76562900 & -24.21034700 & 10.46937300 \\
\hline $\mathrm{H}$ & 0.65253500 & -25.04972500 & 10.01742800 \\
\hline $\mathrm{O}$ & -0.26915700 & -23.55751300 & 7.77672900 \\
\hline
\end{tabular}




$\begin{array}{llll}\mathrm{H} & -0.11854000 & -24.50555900 & 7.78781800 \\ \mathrm{O} & 3.95834200 & -21.99957500 & 8.18069900 \\ \mathrm{H} & 3.90878000 & -21.78721200 & 7.24579500 \\ \mathrm{O} & 0.89459800 & -20.23334800 & 5.77125500 \\ \mathrm{H} & 0.43215800 & -20.43223200 & 4.95382300 \\ \mathrm{O} & 5.01019200 & -17.95407000 & 6.31009200 \\ \mathrm{H} & 4.34573100 & -17.63112400 & 5.69707000 \\ \mathrm{O} & 6.70061500 & -21.20729000 & 10.30834600 \\ \mathrm{H} & 6.94035700 & -21.28210500 & 9.38177800 \\ \mathrm{O} & 7.16071400 & -18.37382900 & 11.27735800 \\ \mathrm{H} & 7.91744600 & -18.32615100 & 10.68856000 \\ \mathrm{O} & 3.04573700 & -12.65259400 & 8.56184100 \\ \mathrm{H} & 3.28141500 & -11.77943000 & 8.23990800 \\ \mathrm{O} & 0.52503600 & -11.03998800 & 8.77301700 \\ \mathrm{H} & 1.19136900 & -10.40469200 & 8.50099000 \\ \mathrm{O} & 0.09532500 & -12.96578500 & 5.93324100 \\ \mathrm{H} & -0.56397300 & -12.45144700 & 5.46166500 \\ \mathrm{O} & -6.48939000 & -14.15705100 & 9.61657100 \\ \mathrm{H} & -6.80928300 & -13.25605600 & 9.53010300 \\ \mathrm{Cl} & 8.35138000 & -21.95395700 & 13.91004000 \\ \mathrm{H} & 0.25005700 & -16.14614200 & 15.30181100\end{array}$

\section{Cartesian coordinates of $1(\AA)$ on Site B}

$\begin{array}{llcc}\text { C } & -7.43176400 & 15.26573500 & 16.29361300 \\ \mathrm{C} & -6.31344200 & 15.72537700 & 15.58776200 \\ \mathrm{C} & -7.40827000 & 14.03592600 & 16.95853300 \\ \mathrm{C} & -5.15677500 & 14.94409100 & 15.60190300 \\ \mathrm{H} & -6.34663300 & 16.65925800 & 15.04234400 \\ \mathrm{C} & -6.24367200 & 13.27462700 & 16.91064500 \\ \mathrm{H} & -8.28738700 & 13.68693700 & 17.48532100 \\ \mathrm{~N} & -5.17170200 & 13.76449000 & 16.25586000 \\ \mathrm{C} & -3.85998300 & 15.24391000 & 14.96327700 \\ \mathrm{C} & -6.03019100 & 11.93234200 & 17.48090500 \\ \mathrm{Pd} & -3.56325700 & 12.66032800 & 16.19806400 \\ \mathrm{C} & -3.57674500 & 16.41914400 & 14.27638800 \\ \mathrm{~N} & -2.90473700 & 14.26942500 & 15.10777500 \\ \mathrm{C} & -7.01245800 & 11.20281300 & 18.14089800 \\ \mathrm{~N} & -4.77680900 & 11.41264900 & 17.29100300 \\ \mathrm{C} & -2.29895400 & 16.60498300 & 13.74277600 \\ \mathrm{H} & -4.33552200 & 17.18451400 & 14.16411800 \\ \mathrm{C} & -1.68612200 & 14.44252200 & 14.58065300 \\ \mathrm{C} & -6.70883900 & 9.92531400 & 18.61579900 \\ \mathrm{H} & -8.00390600 & 11.61910500 & 18.27573700 \\ \mathrm{C} & -4.48619000 & 10.18972900 & 17.74984500 \\ \mathrm{C} & -1.34496600 & 15.60368700 & 13.88999000 \\ \mathrm{H} & -2.06838000 & 17.51687700 & 13.20582100 \\ \mathrm{H} & -0.98228700 & 13.63211400 & 14.72643300 \\ \mathrm{C} & -5.43078200 & 9.41283200 & 18.42073800 \\ \mathrm{H} & -7.46779900 & 9.34361700 & 19.12869000 \\ \mathrm{H} & -3.47854500 & 9.83652700 & 17.56724100 \\ \mathrm{H} & -0.34493900 & 15.70763800 & 13.48411300 \\ \mathrm{H} & -5.15357700 & 8.42568600 & 18.77332800 \\ \mathrm{C} & -8.70093300 & 16.06046100 & 16.35731000 \\ \mathrm{O} & -8.67559600 & 17.13874700 & 15.56009800 \\ \mathrm{O} & -9.64726700 & 15.76214400 & 17.06023400 \\ \mathrm{O} & -11.02021800 & 13.29904300 & 14.46082500 \\ \mathrm{H} & -11.84154200 & 13.41068800 & 13.95552700 \\ \mathrm{O} & -12.96312000 & 16.76519000 & 14.70095700\end{array}$




\begin{tabular}{|c|c|c|c|}
\hline $\mathrm{H}$ & -13.83029600 & 16.34866000 & 14.57287900 \\
\hline $\mathrm{O}$ & -9.47260200 & 21.70824000 & 12.29218800 \\
\hline $\mathrm{H}$ & -9.86692900 & 22.29764700 & 12.95092100 \\
\hline $\mathrm{O}$ & -6.00697100 & 11.61415000 & 14.35907000 \\
\hline $\mathrm{H}$ & -5.78074300 & 12.46166000 & 13.94992200 \\
\hline $\mathrm{Si}$ & -9.70179600 & 13.29530400 & 13.46454300 \\
\hline $\mathrm{Si}$ & -12.25019100 & 17.20004700 & 13.27742500 \\
\hline $\mathrm{Si}$ & -10.42713000 & 21.38183300 & 10.98286400 \\
\hline $\mathrm{Si}$ & -7.48662600 & 11.02776000 & 13.91300400 \\
\hline $\mathrm{Si}$ & -7.07117300 & 14.34741400 & 9.70218700 \\
\hline $\mathrm{Si}$ & -13.03883500 & 19.84266800 & 11.69731200 \\
\hline $\mathrm{Si}$ & -4.94822000 & 17.43406500 & 7.53236000 \\
\hline $\mathrm{Si}$ & -13.80659400 & 14.92479800 & 11.75444500 \\
\hline $\mathrm{Si}$ & -7.01806400 & 17.29751300 & 11.08200600 \\
\hline $\mathrm{Si}$ & -3.81239400 & 14.24992200 & 9.69573800 \\
\hline $\mathrm{Si}$ & -10.98016600 & 16.16684700 & 9.92222100 \\
\hline $\mathrm{Si}$ & -3.99574700 & 17.45005300 & 10.42453600 \\
\hline $\mathrm{Si}$ & -10.08786300 & 13.21334900 & 10.43290600 \\
\hline $\mathrm{Si}$ & -9.39319200 & 16.39983300 & 12.65709000 \\
\hline $\mathrm{Si}$ & -8.07169700 & 19.77281800 & 9.21245800 \\
\hline $\mathrm{O}$ & -9.33525000 & 20.75562700 & 9.84530400 \\
\hline $\mathrm{O}$ & -10.23083900 & 12.77969200 & 12.01012700 \\
\hline $\mathrm{O}$ & -8.61858800 & 12.24950800 & 14.18690700 \\
\hline $\mathrm{O}$ & -2.71307000 & 18.35500000 & 11.15790000 \\
\hline $\mathrm{O}$ & -14.41730000 & 15.15500000 & 10.22270000 \\
\hline $\mathrm{O}$ & -6.73871000 & 20.65170000 & 8.75008000 \\
\hline $\mathrm{O}$ & -13.87740000 & 19.02240000 & 10.57480000 \\
\hline $\mathrm{O}$ & -11.08734700 & 14.49705400 & 10.04182400 \\
\hline $\mathrm{O}$ & -4.50329900 & 18.08573900 & 8.98382900 \\
\hline $\mathrm{O}$ & -6.01620000 & 16.17470000 & 7.83313000 \\
\hline $\mathrm{O}$ & -12.80096200 & 18.73047000 & 12.92468600 \\
\hline $\mathrm{O}$ & -13.25740000 & 13.34730000 & 11.94560000 \\
\hline $\mathrm{O}$ & -7.32277000 & 13.88160000 & 8.14201000 \\
\hline $\mathrm{O}$ & -12.61034000 & 15.99623400 & 12.16609000 \\
\hline $\mathrm{O}$ & -11.48325200 & 22.76524000 & 10.88106100 \\
\hline $\mathrm{O}$ & -7.18966600 & 15.88083100 & 10.27684100 \\
\hline $\mathrm{O}$ & -7.61763400 & 18.63507600 & 10.32478300 \\
\hline $\mathrm{O}$ & -10.02495400 & 16.56209800 & 11.17546500 \\
\hline $\mathrm{O}$ & -14.93290000 & 15.23010000 & 12.93830000 \\
\hline $\mathrm{O}$ & -10.59512100 & 17.16618700 & 13.55288500 \\
\hline $\mathrm{O}$ & -10.04340000 & 16.49330000 & 8.64193000 \\
\hline $\mathrm{O}$ & -9.15045400 & 14.86701000 & 13.25917300 \\
\hline $\mathrm{O}$ & -7.89221600 & 17.11917000 & 12.51350800 \\
\hline $\mathrm{O}$ & -5.37128700 & 17.49990300 & 11.39214800 \\
\hline $\mathrm{O}$ & -12.29090000 & 17.20570000 & 9.76648000 \\
\hline $\mathrm{O}$ & -5.43904500 & 13.89498300 & 9.99551000 \\
\hline $\mathrm{O}$ & -10.75460000 & 11.87220000 & 9.63893000 \\
\hline $\mathrm{O}$ & -8.45557400 & 13.59593200 & 10.30061800 \\
\hline $\mathrm{O}$ & -11.49916100 & 20.17315700 & 11.25426200 \\
\hline $\mathrm{O}$ & -7.62428800 & 10.99042100 & 12.23680400 \\
\hline $\mathrm{O}$ & -6.05862000 & 18.41460000 & 6.70938000 \\
\hline $\mathrm{O}$ & -8.36776000 & 19.04050000 & 7.77424000 \\
\hline $\mathrm{O}$ & -14.03870000 & 21.24120000 & 11.80930000 \\
\hline $\mathrm{O}$ & -3.58534800 & 15.86339900 & 10.10627000 \\
\hline $\mathrm{H}$ & -14.01376800 & 12.76123600 & 11.86790600 \\
\hline $\mathrm{H}$ & -15.85316300 & 15.03888000 & 12.74297500 \\
\hline $\mathrm{H}$ & -14.94428600 & 14.44996800 & 9.83953400 \\
\hline $\mathrm{H}$ & -10.50013200 & 11.85484200 & 8.71343300 \\
\hline
\end{tabular}




$\begin{array}{lccc}\mathrm{H} & -7.06179500 & 10.35582300 & 11.78681600 \\ \mathrm{H} & -6.53214100 & 13.78211100 & 7.60665300 \\ \mathrm{H} & -6.23155900 & 15.64069300 & 7.06497800 \\ \mathrm{H} & -5.85669400 & 18.27574800 & 5.78118500 \\ \mathrm{H} & -7.97560700 & 19.43408500 & 6.99135600 \\ \mathrm{H} & -7.01381400 & 21.32421300 & 8.12267000 \\ \mathrm{H} & -12.62072400 & 17.34281800 & 8.87540500 \\ \mathrm{H} & -11.71078800 & 22.88197300 & 9.95578200 \\ \mathrm{H} & -14.52986200 & 21.26959200 & 10.98494900 \\ \mathrm{H} & -14.64947900 & 19.53628300 & 10.32697400 \\ \mathrm{O} & -7.45726200 & 9.56086300 & 14.86485500 \\ \mathrm{H} & -7.84325200 & 8.81679500 & 14.39693700 \\ \mathrm{O} & -2.74946700 & 13.39743500 & 10.82646300 \\ \mathrm{H} & -1.96632400 & 13.11215900 & 10.35011900 \\ \mathrm{O} & -3.45515800 & 13.93857100 & 7.96439700 \\ \mathrm{H} & -2.60552500 & 13.49410400 & 7.91778200 \\ \mathrm{O} & -3.41612800 & 17.09429000 & 6.78684100 \\ \mathrm{H} & -3.27427500 & 17.72712400 & 6.07902700 \\ \mathrm{H} & -2.03555200 & 18.76203900 & 10.61302500 \\ \mathrm{H} & -10.40411500 & 16.21904900 & 7.79560200 \\ \mathrm{Cl} & -1.62867300 & 11.32768600 & 16.12510500 \\ \mathrm{H} & -9.56995000 & 17.53064500 & 15.52733200\end{array}$

\begin{tabular}{lccc}
\multicolumn{5}{c}{ Cartesian coordinates of $\mathbf{1}(\mathbf{\AA})$ on Site C } \\
$\mathrm{C}$ & -9.25078900 & 6.34251300 & 17.32393500 \\
$\mathrm{C}$ & -8.22990200 & 5.39899400 & 17.48117800 \\
$\mathrm{C}$ & -10.59006200 & 6.00159600 & 17.54063800 \\
$\mathrm{C}$ & -8.57743700 & 4.09863000 & 17.84343300 \\
$\mathrm{H}$ & -7.19662500 & 5.67140000 & 17.30697700 \\
$\mathrm{C}$ & -10.88743600 & 4.68204200 & 17.87816500 \\
$\mathrm{H}$ & -11.37775000 & 6.72943200 & 17.40365400 \\
$\mathrm{~N}$ & -9.88094000 & 3.79636700 & 18.01052900 \\
$\mathrm{C}$ & -7.67252000 & 2.95181600 & 18.04384400 \\
$\mathrm{C}$ & -12.22775500 & 4.08766700 & 18.03967100 \\
$\mathrm{Pd}$ & -10.34604400 & 1.93111300 & 18.33749600 \\
$\mathrm{C}$ & -6.28722800 & 3.02471200 & 17.94901500 \\
$\mathrm{~N}$ & -8.29651500 & 1.76701500 & 18.33465300 \\
$\mathrm{C}$ & -13.41556900 & 4.80776800 & 17.95854200 \\
$\mathrm{~N}$ & -12.23848000 & 2.73129900 & 18.24364200 \\
$\mathrm{C}$ & -5.52948200 & 1.86929500 & 18.15264600 \\
$\mathrm{H}$ & -5.80328900 & 3.96773500 & 17.72177000 \\
$\mathrm{C}$ & -7.56931000 & 0.66202400 & 18.53286000 \\
$\mathrm{C}$ & -14.63197400 & 4.13057700 & 18.07940400 \\
$\mathrm{H}$ & -13.39705700 & 5.88086200 & 17.80576100 \\
$\mathrm{C}$ & -13.40491400 & 2.08570400 & 18.36254800 \\
$\mathrm{C}$ & -6.17660600 & 0.67449900 & 18.44920700 \\
$\mathrm{H}$ & -4.44769400 & 1.91147200 & 18.08010400 \\
$\mathrm{H}$ & -8.12229200 & -0.24230300 & 18.75635400 \\
$\mathrm{C}$ & -14.62721700 & 2.75447400 & 18.28140400 \\
$\mathrm{H}$ & -15.56557600 & 4.67978900 & 18.01706000 \\
$\mathrm{H}$ & -13.34599000 & 1.01600100 & 18.52352000 \\
$\mathrm{H}$ & -5.62590800 & -0.24439700 & 18.61651400 \\
$\mathrm{H}$ & -15.54829800 & 2.19125600 & 18.38028700 \\
$\mathrm{C}$ & -8.88815500 & 7.69369400 & 16.80866100 \\
$\mathrm{O}$ & -7.75947900 & 7.97464800 & 16.41229000 \\
$\mathrm{O}$ & -9.89882100 & 8.55416300 & 16.77993800 \\
$\mathrm{O}$ & -12.68343000 & 7.40596900 & 13.71851100
\end{tabular}




\begin{tabular}{|c|c|c|c|}
\hline $\mathrm{H}$ & -12.90645300 & 6.93066300 & 14.53241700 \\
\hline $\mathrm{O}$ & -5.12260600 & 11.87259500 & 14.28296900 \\
\hline $\mathrm{H}$ & -4.79861100 & 12.67947700 & 14.70668600 \\
\hline $\mathrm{O}$ & -11.04020600 & 13.42165400 & 14.94040600 \\
\hline $\mathrm{H}$ & -11.54703400 & 12.60693100 & 15.13218100 \\
\hline $\mathrm{O}$ & -5.99941200 & 7.12542000 & 14.46464800 \\
\hline $\mathrm{H}$ & -6.65933200 & 7.12016700 & 15.17641400 \\
\hline $\mathrm{O}$ & -12.33774700 & 11.41407000 & 14.37902400 \\
\hline $\mathrm{H}$ & -11.76518100 & 10.65199600 & 14.21760400 \\
\hline $\mathrm{O}$ & -12.15671900 & 5.35620100 & 14.87265900 \\
\hline $\mathrm{H}$ & -12.35448400 & 4.50143300 & 15.28326300 \\
\hline $\mathrm{O}$ & -6.89233500 & 10.32929400 & 14.91271200 \\
\hline $\mathrm{H}$ & -7.14264100 & 9.53155500 & 15.40684700 \\
\hline $\mathrm{Si}$ & -13.59945400 & 6.94542200 & 12.40963300 \\
\hline $\mathrm{Si}$ & -4.25214000 & 11.47808200 & 12.96229500 \\
\hline $\mathrm{Si}$ & -9.92143000 & 13.28827900 & 13.76059000 \\
\hline $\mathrm{Si}$ & -3.57889800 & 8.35091200 & 12.23109200 \\
\hline $\mathrm{O}$ & -5.12693900 & 7.63151300 & 12.06831100 \\
\hline $\mathrm{Si}$ & -6.44582200 & 6.94763300 & 12.89957900 \\
\hline $\mathrm{Si}$ & -9.70325600 & 2.39032100 & 14.01736700 \\
\hline $\mathrm{Si}$ & -13.08447100 & 12.04381200 & 12.98262800 \\
\hline $\mathrm{Si}$ & -9.37406700 & 8.07389000 & 13.33743900 \\
\hline $\mathrm{O}$ & -9.12942100 & 9.51119000 & 14.14478300 \\
\hline $\mathrm{Si}$ & -10.75713700 & 5.30113800 & 13.98055900 \\
\hline $\mathrm{O}$ & -9.78842000 & 6.65899600 & 14.18138500 \\
\hline $\mathrm{Si}$ & -8.03987200 & 10.77781400 & 13.83122500 \\
\hline $\mathrm{Si}$ & -7.31495300 & 3.79896300 & 12.21492200 \\
\hline $\mathrm{Si}$ & -11.88377400 & 9.24290200 & 11.65383600 \\
\hline $\mathrm{O}$ & -6.81684400 & 5.33379900 & 12.64442000 \\
\hline $\mathrm{O}$ & -10.29020100 & 12.77539800 & 12.16160100 \\
\hline $\mathrm{O}$ & -8.75095500 & 12.22653900 & 14.25608900 \\
\hline $\mathrm{O}$ & -10.23274200 & 3.85455400 & 14.60739000 \\
\hline $\mathrm{O}$ & -8.58243500 & 3.03563100 & 12.96693200 \\
\hline $\mathrm{O}$ & -6.14375500 & 2.69370700 & 12.57229200 \\
\hline $\mathrm{O}$ & -3.08446400 & 12.68769300 & 12.97730600 \\
\hline $\mathrm{O}$ & -10.93353300 & 5.14357700 & 12.29792900 \\
\hline $\mathrm{O}$ & -12.58531200 & 13.53519400 & 12.16053500 \\
\hline $\mathrm{O}$ & -2.80701200 & 7.41188400 & 13.34626600 \\
\hline $\mathrm{O}$ & -12.69444700 & 10.66656000 & 12.04994900 \\
\hline $\mathrm{O}$ & -13.43625600 & 5.41706500 & 11.71461200 \\
\hline $\mathrm{O}$ & -5.21976300 & 11.60125000 & 11.60259600 \\
\hline $\mathrm{O}$ & -7.91723300 & 7.72019100 & 12.60301500 \\
\hline $\mathrm{O}$ & -9.70342200 & 14.91180100 & 13.52970200 \\
\hline $\mathrm{O}$ & -3.70934000 & 9.91100500 & 12.85184600 \\
\hline $\mathrm{O}$ & -13.08558400 & 8.13275500 & 11.35671800 \\
\hline $\mathrm{O}$ & -7.62421400 & 10.99057100 & 12.23679700 \\
\hline $\mathrm{O}$ & -15.13139800 & 7.14424600 & 13.04146900 \\
\hline $\mathrm{O}$ & -10.69514300 & 8.53362100 & 12.53009800 \\
\hline $\mathrm{O}$ & -10.91580700 & 1.48717300 & 13.05084100 \\
\hline $\mathrm{H}$ & -10.88820100 & 0.57978000 & 13.36305200 \\
\hline $\mathrm{O}$ & -9.08682000 & 1.59154300 & 15.48352700 \\
\hline $\mathrm{H}$ & -9.43404000 & 0.69656700 & 15.49098700 \\
\hline $\mathrm{O}$ & -7.70935400 & 3.98482000 & 10.59926300 \\
\hline $\mathrm{H}$ & -7.32352500 & 3.23794700 & 10.13567200 \\
\hline $\mathrm{O}$ & -2.58185200 & 8.40065000 & 10.82942900 \\
\hline $\mathrm{H}$ & -1.64168100 & 8.30502100 & 10.99834800 \\
\hline $\mathrm{H}$ & -5.27038900 & 3.08706100 & 12.50821900 \\
\hline $\mathrm{H}$ & -2.26565400 & 12.18841200 & 13.02033700 \\
\hline
\end{tabular}




$\begin{array}{cccc}\mathrm{H} & -4.56068700 & 11.61532600 & 10.90473700 \\ \mathrm{H} & -6.72017200 & 10.97392900 & 11.91421800 \\ \mathrm{H} & -8.75209900 & 15.03958600 & 13.51358800 \\ \mathrm{H} & -9.43959300 & 12.65020500 & 11.73452400 \\ \mathrm{H} & -11.63362000 & 13.63713100 & 12.08634800 \\ \mathrm{H} & -12.52058100 & 5.19602200 & 11.52870300 \\ \mathrm{H} & -15.17630900 & 7.98078300 & 13.51027300 \\ \mathrm{O} & -14.63462600 & 12.19585300 & 13.78299800 \\ \mathrm{H} & -15.07856700 & 13.01537300 & 13.55297800 \\ \mathrm{O} & -10.95016000 & 9.44878900 & 10.30917700 \\ \mathrm{H} & -11.68093500 & 9.58957800 & 9.70275600 \\ \mathrm{H} & -1.85599600 & 7.59741600 & 13.45224300 \\ \mathrm{H} & -10.03043700 & 5.08643700 & 11.97643900 \\ \mathrm{Cl} & -10.90526100 & -0.32748300 & 18.63900400 \\ \mathrm{H} & -9.63398600 & 9.34115800 & 16.26134300\end{array}$

\section{Cartesian coordinates of 1 (̊) on Site D}

$\begin{array}{lccc}\mathrm{C} & 11.03477300 & -7.79287300 & 15.36775700 \\ \mathrm{C} & 12.19267500 & -8.52895300 & 15.09385300 \\ \mathrm{C} & 9.85709900 & -8.42974800 & 15.77420800 \\ \mathrm{C} & 12.14247800 & -9.91858000 & 15.22750600 \\ \mathrm{H} & 13.09738000 & -8.03545900 & 14.76469400 \\ \mathrm{C} & 9.85619400 & -9.81768400 & 15.89057300 \\ \mathrm{H} & 8.96602100 & -7.84694900 & 15.96460200 \\ \mathrm{~N} & 10.98697700 & -10.49689400 & 15.60873600 \\ \mathrm{C} & 13.22759900 & -10.88429200 & 14.97117000 \\ \mathrm{C} & 8.71260900 & -10.68623600 & 16.22843500 \\ \mathrm{Pd} & 10.93926300 & -12.44357800 & 15.75142800 \\ \mathrm{C} & 14.50891400 & -10.53001700 & 14.56634000 \\ \mathrm{~N} & 12.88446100 & -12.20110100 & 15.14205900 \\ \mathrm{C} & 7.42058500 & -10.21983300 & 16.44604500 \\ \mathrm{~N} & 8.98801800 & -12.02904300 & 16.24534300 \\ \mathrm{C} & 15.44919100 & -11.53389400 & 14.32239200 \\ \mathrm{H} & 14.76988800 & -9.48696900 & 14.43303400 \\ \mathrm{C} & 13.78171100 & -13.16298900 & 14.89805100 \\ \mathrm{C} & 6.39346800 & -11.14054900 & 16.66948300 \\ \mathrm{H} & 7.21113200 & -9.15780600 & 16.42439300 \\ \mathrm{C} & 8.00504200 & -12.90859200 & 16.46811000 \\ \mathrm{C} & 15.08025300 & -12.86608100 & 14.48123200 \\ \mathrm{H} & 16.45322000 & -11.27050900 & 14.00596600 \\ \mathrm{H} & 13.44406500 & -14.18193600 & 15.04353500 \\ \mathrm{C} & 6.68783100 & -12.49956200 & 16.68082200 \\ \mathrm{H} & 5.37835500 & -10.79097800 & 16.82720700 \\ \mathrm{H} & 8.28758400 & -13.95438400 & 16.46435800 \\ \mathrm{H} & 15.77722500 & -13.67553400 & 14.29546600 \\ \mathrm{H} & 5.91958700 & -13.24637300 & 16.84645000 \\ \mathrm{C} & 10.97985400 & -6.31010800 & 15.15252300 \\ \mathrm{O} & 12.19038300 & -5.73978400 & 15.05351900 \\ \mathrm{O} & 9.93498400 & -5.69411600 & 15.06567600 \\ \mathrm{O} & 13.77246600 & -10.80647200 & 11.48224000 \\ \mathrm{H} & 14.24938000 & -11.61838200 & 11.70618200 \\ \mathrm{O} & 8.85217200 & -11.31260300 & 13.24782900 \\ \mathrm{O} & 7.53675600 & -8.44934000 & 13.64153500 \\ \mathrm{Si} & 9.83127100 & -12.31607300 & 12.27347100 \\ \mathrm{Si} & 12.18943400 & -10.96199900 & 11.07300500 \\ \mathrm{Si} & 12.44671200 & -5.37311500 & 11.72308900 \\ \mathrm{O} & 11.76851600 & -4.20707100 & 12.74244500 \\ \mathrm{Si} & 6.79610100 & -7.07122300 & 12.95012200\end{array}$




\begin{tabular}{|c|c|c|c|}
\hline $\mathrm{Si}$ & 10.90756300 & -2.75154400 & 12.61654600 \\
\hline $\mathrm{Si}$ & 8.21093900 & -9.76627100 & 12.75826000 \\
\hline $\mathrm{Si}$ & 7.84358800 & -4.22631800 & 12.00402200 \\
\hline $\mathrm{Si}$ & 10.84424300 & -8.14453900 & 11.57306300 \\
\hline $\mathrm{Si}$ & 2.63679700 & -10.44283900 & 12.71531800 \\
\hline $\mathrm{Si}$ & 8.61033700 & -12.05828800 & 9.35828600 \\
\hline $\mathrm{Si}$ & 3.61180300 & -7.39298700 & 12.94538100 \\
\hline $\mathrm{Si}$ & 5.40420900 & -10.67546500 & 11.18613500 \\
\hline $\mathrm{Si}$ & 5.96105700 & -13.59672700 & 9.71559500 \\
\hline $\mathrm{Si}$ & 10.27764200 & -15.26976200 & 11.10440700 \\
\hline $\mathrm{Si}$ & 4.12573400 & -7.94982500 & 9.97427400 \\
\hline $\mathrm{Si}$ & 8.37085300 & -6.69778500 & 10.40378200 \\
\hline $\mathrm{Si}$ & 11.35719700 & -13.12505100 & 8.82825100 \\
\hline $\mathrm{O}$ & 5.20663900 & -7.14185700 & 13.49911600 \\
\hline $\mathrm{O}$ & 3.04764000 & -8.91847300 & 13.29147600 \\
\hline $\mathrm{O}$ & 9.90403700 & -7.22983500 & 10.62611700 \\
\hline $\mathrm{O}$ & 8.04770000 & -7.22359000 & 8.92560000 \\
\hline $\mathrm{O}$ & 2.56959000 & -8.29680000 & 9.65349000 \\
\hline $\mathrm{O}$ & 10.96779300 & -14.45772400 & 9.78327300 \\
\hline $\mathrm{O}$ & 12.09008500 & -11.93933000 & 9.73062500 \\
\hline $\mathrm{O}$ & 14.12480000 & -5.05663000 & 11.49480000 \\
\hline $\mathrm{O}$ & 4.14338100 & -10.98249300 & 12.24505400 \\
\hline $\mathrm{O}$ & 11.92728700 & -6.95361900 & 12.18688600 \\
\hline $\mathrm{O}$ & 7.54452600 & -13.31052900 & 9.28661000 \\
\hline $\mathrm{O}$ & 2.36335000 & -6.39464000 & 13.52040000 \\
\hline $\mathrm{O}$ & 11.36722500 & -11.69467900 & 12.31605800 \\
\hline $\mathrm{O}$ & 7.16681500 & -7.34308400 & 11.35162700 \\
\hline $\mathrm{O}$ & 6.85549600 & -10.29424400 & 11.93032500 \\
\hline $\mathrm{O}$ & 11.81300000 & -5.09078000 & 10.26070000 \\
\hline $\mathrm{O}$ & 9.68127800 & -9.01468900 & 12.41305100 \\
\hline $\mathrm{O}$ & 9.88504300 & -12.46127100 & 8.34113200 \\
\hline $\mathrm{O}$ & 4.99007900 & -9.36077900 & 10.24741400 \\
\hline $\mathrm{O}$ & 8.34164100 & -5.06679000 & 10.66303900 \\
\hline $\mathrm{O}$ & 9.26777800 & -11.91814400 & 10.81652400 \\
\hline $\mathrm{O}$ & 9.96313000 & -14.01810300 & 12.25242700 \\
\hline $\mathrm{O}$ & 4.63544000 & -6.90307000 & 8.78162000 \\
\hline $\mathrm{O}$ & 9.23309200 & -3.25018900 & 12.42526100 \\
\hline $\mathrm{O}$ & 6.35722000 & -3.39951000 & 11.58530000 \\
\hline $\mathrm{O}$ & 7.85965000 & -10.54490000 & 9.22149000 \\
\hline $\mathrm{O}$ & 11.70750400 & -9.37777400 & 10.86014900 \\
\hline $\mathrm{O}$ & 5.50978300 & -12.14228900 & 10.40321800 \\
\hline $\mathrm{O}$ & 3.92502800 & -7.10468400 & 11.36378000 \\
\hline $\mathrm{O}$ & 7.37473900 & -5.46317500 & 13.10318600 \\
\hline $\mathrm{H}$ & 14.23096700 & -4.75901400 & 10.58829400 \\
\hline $\mathrm{H}$ & 12.41078600 & -4.91235900 & 9.53103000 \\
\hline $\mathrm{H}$ & 6.52156600 & -2.58036400 & 11.11244700 \\
\hline $\mathrm{H}$ & 1.76962000 & -6.09167700 & 12.82953200 \\
\hline $\mathrm{H}$ & 2.48429100 & -8.69595100 & 8.78458100 \\
\hline $\mathrm{H}$ & 4.12413300 & -6.89248100 & 7.96918300 \\
\hline $\mathrm{H}$ & 7.52313800 & -10.62821900 & 8.32627100 \\
\hline $\mathrm{H}$ & 8.70573500 & -7.37989400 & 8.24430800 \\
\hline $\mathrm{O}$ & 11.53771200 & -16.49589700 & 11.60675300 \\
\hline $\mathrm{H}$ & 11.74049800 & -17.02117900 & 10.82922100 \\
\hline $\mathrm{O}$ & 8.68306000 & -15.95030700 & 10.72472300 \\
\hline $\mathrm{H}$ & 8.71540800 & -16.26500800 & 9.81834700 \\
\hline $\mathrm{O}$ & 12.37106900 & -13.52105400 & 7.45737000 \\
\hline $\mathrm{H}$ & 11.69261300 & -13.80020400 & 6.83819900 \\
\hline $\mathrm{O}$ & 11.15532800 & -1.90116400 & 14.21691500 \\
\hline
\end{tabular}


$\mathrm{H} \quad \begin{array}{llll}11.52518300 & -1.02949900 & 14.05877600\end{array}$

$\begin{array}{llll}\mathrm{O} & 11.65594700 & -1.87723900 & 11.20508800\end{array}$

$\mathrm{H} \quad 11.89532000 \quad-0.98634400 \quad 11.47080600$

$\begin{array}{llll}\mathrm{O} & 1.95327500 & -11.51804200 & 14.01077000\end{array}$

$\mathrm{H} \quad 1.00560800 \quad-11.55475900 \quad 13.86184300$

$\begin{array}{llll}\mathrm{O} & 1.65039500 & -10.20918200 & 11.29570200\end{array}$

H $\quad \begin{array}{lllll}0.77752800 & -10.56180500 & 11.48374500\end{array}$

O $\quad 5.89312500 \quad-14.98325600 \quad 10.84658500$

$\mathrm{H} \quad 5.31586900 \quad-15.63225300 \quad 10.43771000$

$\begin{array}{lllll}\mathrm{O} & 5.13503200 & -13.74326700 & 8.17890300\end{array}$

$\mathrm{H} \quad 4.84731300 \quad-14.65450400 \quad 8.08689800$

$\mathrm{Cl} \quad 10.90454600 \quad-14.77844900 \quad 15.97137500$

$\begin{array}{llll}\mathrm{H} & 12.06071400 & -4.83460900 & 14.70392800\end{array}$ 